\title{
Higher Spin Gauge Theories in Various Dimensions
}

\author{
Mikhail Vasiliev* \\ Lebedev Physical Institute \\ E-mail: vasiliev@lpi.ru
}

ABSTRACT: Properties of nonlinear higher spin gauge theories of totally symmetric massless higher spin fields in anti-de Sitter space of any dimension are discussed with the emphasize on the general aspects of the approach.

\section{Introduction}

As shown by Fronsdal [1], an integer-spin massless spin- $s$ field is described by a totally symmetric tensor $\varphi_{n_{1} \ldots n_{s}}(m, n, \ldots=0, \ldots, d-1$ are $d$-dimensional vector indices) subject to the double tracelessness condition $\varphi^{r}{ }_{r}{ }_{k n} n_{5} \ldots n_{s}=0$ which is nontrivial for $s \geq 4$. The quadratic action for a free spin $s$ field $\varphi_{n_{1} \ldots n_{s}}$ is fixed up to an overall factor in the form $S_{s}=$ $\varphi L \varphi$ with some second order differential operator $L$ by the condition of gauge invariance under the Abelian gauge transformations $\delta \varphi_{n_{1} \ldots n_{s}}=\partial_{\left\{n_{1}\right.} \varepsilon_{\left.n_{2} \ldots n_{s}\right\}}$ with symmetric traceless tensor parameters $\varepsilon_{n_{1} \ldots n_{s-1}}, \varepsilon_{r n_{3} \ldots n_{s-1}}^{r}=0$. It is the higher spin (HS) gauge symmetry principle that makes the HS gauge theories interesting and perhaps fundamental. The free HS gauge theories extend the linearized theories of electromagnetism (spin 1) and gravity (spin 2) in a uniform way. The original Fronsdal theory and its more geometric versions [2, 3] generalize the metric formulation of gravity. The HS generalization of Cartan formulation of gravity with the HS fields described in terms of the frame-like 1-forms was

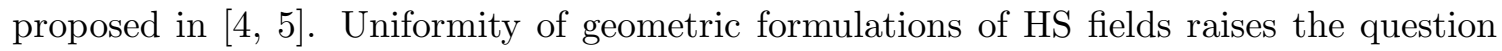
whether there exists an underlying nonlinear HS gauge theory which in the free field limit gives rise to the free Fronsdal Lagrangians. There is a number of motivations for studying HS gauge theories.

From supergravity perspective, this is interesting because theories with HS fields may have more supersymmetries than the "maximal" supergravities with 32 supercharges. Recall that the limitation that the number of supercharges is $\leq 32$ is a direct consequence of the requirement that $s \leq 2$ for all fields in a supermultiplet (see e.g. [6]). From superstring perspective, the most obvious motivation is due to Stueckelberg symmetries in the string

\footnotetext{
${ }^{*}$ Speaker.
} 
field theory [7], which have a form of spontaneously broken HS gauge symmetries. An important indication in the same direction is that string amplitudes exhibit certain symmetries in the high-energy limit equivalent to the string mass parameter tending to zero [8.

Unusual feature of interacting HS gauge theories is that unbroken HS gauge symmetries do not allow flat space-time as a vacuum solution, requiring nonzero curvature [9]. Anti-de Sitter $(A d S)$ space is the most symmetric vacuum of this type. This property may admit interpretation [10, 11, 12] in the context of the $A d S / C F T$ correspondence conjecture [13]. In particular, it was conjectured [10, 11] that HS gauge theories in AdS bulk are dual to some conformal models on the AdS boundary in the large $N$ limit with $g^{2} N \rightarrow 0$ where $g^{2}$ is the boundary coupling constant. Again, this indicates that HS gauge theory has a good chance to be related to a symmetric phase of superstring theory. On the other hand, a reason why the HS gauge theory may be hard to observe in superstring theory may be that a quantum formulation of the latter is not still available in the AdS background despite the progress achieved at the classical level [14].

Whatever a motivation is, the HS problem is to find any nonlinear theory such that

- In the free field limit it contains a set of Fronsdal fields (with correct signs of kinetic terms) plus, may be, some other fields that admit consistent quantization (e.g., mixed symmetry massless fields which exist in $d>4[15]$ ).

- HS gauge symmetries are unbroken in a nonlinear HS theory and are deformed to some non-Abelian symmetry.

The first condition rules out ghosts ${ }^{1}$. The condition that a HS symmetry is non-Abelian avoids a trivial possibility of building a nonlinear theory with undeformed Abelian HS symmetries by adding powers of gauge invariant HS field strengths to the Fronsdal action like, for example, adding powers of the Maxwell field strengths to a collection of free Maxwell actions instead of deforming it to the Yang-Mills action. For the HS models we discuss, this condition is satisfied as a result of the manifest invariance under diffeomorphisms. A structure of the HS symmetry is one of the key elements of the theory.

Although being absolutely minimal, these conditions are so restrictive that they were believed for a long period to admit no solution at all. One argument was due to the Coleman-Mandula type no-go theorems [17] which state that any $S$ matrix in flat spacetime, that has a symmetry larger than (semi)direct product of usual space-time (super)symmetries and inner symmetries, is trivial $(S=I d)$. Since no scattering means no interactions, this sounds like no theory with non-Abelian HS global symmetries can exist.

An alternative test was provided by the attempt [18] to introduce interactions of a HS gauge field with gravity. It is straightforward to see that the standard covariantization procedure $\partial \rightarrow D=\partial-\Gamma$ breaks down the invariance under the HS gauge transformations because, in order to prove invariance of the action $S_{s}$, one has to commute derivatives,

\footnotetext{
${ }^{1}$ Note that the standard HS field dynamics is free of ghosts but this is not the case, e.g., for the partially massless HS models of [16] in $A d S$.
} 
while the commutator of the covariant derivatives is proportional to the Riemann tensor, $[D \ldots, D \ldots]=\mathcal{R} \ldots$. As a result, the gauge variation of the covariantized action $S_{s}^{c o v}$ is different from zero, having the structure

$$
\delta S_{s}^{c o v}=\int \mathcal{R} \ldots\left(\varepsilon_{\ldots} D \varphi_{\ldots}\right) \neq 0 .
$$

It seems difficult to cancel these terms because for $s>2$ they contain the Weyl tensor part of $\mathcal{R}$ which cannot be compensated by a transformation of the gravitational field.

On the other hand, a number of indications on the existence of some consistent interactions of the HS fields were found both in the light-cone [19] and in the covariant approach [20], giving strong evidence that some fundamental HS gauge theory must exist. In these works, the problem was considered in flat space. Somewhat later it was realized 9 that the situation improves drastically once the problem is analyzed in the $A d S$ space with nonzero curvature $\Lambda$. This allowed constructing consistent $4 d$ HS-gravitational interactions in the cubic order at the action level [9] and, later, in all orders in interactions at the level of equations of motion [21]. Recently, the $4 d$ results of [21] were generalized to any space-time dimension [22].

The role of $A d S$ background in HS gauge theories is important in many respects. In particular it cancels the Coleman-Mandula argument because $A d S$ space admits no $S$ matrix, and fits naturally the AdS/CFT correspondence conjecture. From the technical side, the dimensionful cosmological constant allows new types of HS interactions with higher derivatives, which resolve the problem with HS-gravitational interactions as follows. The Riemann tensor $\mathcal{R}_{n m, k l}$ is not small near $A d S$ background but

$$
\mathcal{R}_{n m, k l}=R_{n m, k l}-\Lambda\left(g_{n k} g_{m l}-g_{n l} g_{m k}\right),
$$

where $\Lambda$ is the cosmological constant, $g_{m n}$ is the background $A d S$ metric tensor and $R$ is a deviation of the Riemann tensor from the $A d S$ background curvature. Expanding around the AdS geometry is therefore equivalent to expanding in powers of $R$ rather than in powers of the Riemann tensor $\mathcal{R}$. The crucial difference compared to the flat space is that the commutator of covariant derivatives in the $A d S$ space

$$
\left[D_{n}, D_{m}\right] \sim \Lambda
$$

is not small for general $\Lambda$. With nonzero $\Lambda$ one can add to the action some cubic terms schematically written in the form

$$
S^{i n t}=\int \sum_{p, q} \alpha(p, q) \Lambda^{-\frac{1}{2}(p+q)} D^{p}(\varphi) D^{q}(\varphi) R,
$$

which contain higher derivatives ${ }^{2}$ with some of the coefficients proportional to negative powers of $\Lambda$. There exists such a unique (modulo total derivatives and field redefinitions) action correction by the terms (1.4) with a minimal order of derivatives that its HS gauge

\footnotetext{
${ }^{2} D^{p}$ and $D^{q}$ denote here some combinations of derivatives of orders $p$ and $q$, respectively.
} 
variation exactly compensates the original variation (1.1) (for any two given spins an order of minimally necessary derivatives in the vertex (1.4) is finite, increasing linearly with the sum of spins). This implies that $\Lambda$ should necessarily be nonzero in the phase with unbroken HS gauge symmetries. In that respect, HS gauge theories are analogous to gauged supergravities with charged gravitinos, which also require $\Lambda \neq 0$.

Properties of HS gauge theories are to large extent determined by HS global symmetries of their most symmetric vacua. HS symmetry restricts interactions and fixes spectra of spins of massless fields in HS theories as ordinary supersymmetry does in supergravity. To elucidate the structure of a global HS (super)algebra $h$ it is useful to use the approach in which fields, action and transformation laws are formulated in terms of the gauge fields of $h$. An attractive feature of this approach, which generalizes the MacDowell-MansouriStelle-West approach [23, 24] to gravity, is that it treats all fields as differential forms with the gravitational field being on equal footing with other massless fields in a HS multiplet. The only special property of the metric tensor is that it has a nonzero vacuum expectation value allowing a meaningful linearized approximation for all fields in the model. In section 2 we recall the MacDowell-Mansouri-Stelle-West approach to gravity. Then in section 01 we show following to 5 how free HS fields can be reformulated in terms of differential forms to be interpreted as gauge connections. The non-Abelian HS algebra is defined in section t 1 in terms of some star-product algebra. Then we describe unfolded formulation of free field HS dynamics in section 5 and formulate nonlinear HS equations in section 5 . Some conclusions and perspectives are summarized in the Conclusion.

\section{Gravity as $o(d-1,2)$ gauge theory}

Our approach to higher spins generalizes the MacDowell-Mansouri-Stelle-West [23, 24] formulation of gravity as $o(d-1,2)$ gauge theory. The key observation is that the frame 1-form $e^{a}(x)=d x^{n} e_{n}{ }^{a}(x)$ and Lorentz connection $\omega^{a b}(x)=d x^{n} \omega_{n}{ }^{a b}(x)$ can be interpreted as components of the $o(d-1,2)$ connection 1-form $\omega^{A B}(x)=d x^{n} \omega_{n}{ }^{A B}(x)(a, b, \ldots=$ $0, \ldots, d-1$ are fiber Lorentz vector indices and $A, B, \ldots=0, \ldots, d$ are fiber $o(d-1,2)$ vector indices). The Lorentz subalgebra $o(d-1,1) \in o(d-1,2)$ is identified with the stability subalgebra of some vector $V^{A}$. Since Lorentz symmetry is local, this vector can be chosen differently at different points of space-time, thus becoming a field $V^{A}=V^{A}(x)$. It is convenient to relate its norm to the cosmological constant so that $V^{A}$ has dimension of length ${ }^{3}$

$$
V^{A} V_{A}=-\Lambda^{-1}
$$

This allows for a covariant definition of the frame field and Lorentz connection 24

$$
E^{A}=D\left(V^{A}\right) \equiv d V^{A}+\omega^{A B} V_{B}, \quad \omega^{\mathcal{L} A B}=\omega^{A B}+\Lambda\left(E^{A} V^{B}-E^{B} V^{A}\right) .
$$

\footnotetext{
${ }^{3} \Lambda$ is negative and positive in the $A d S$ and $\mathrm{dS}$ cases, respectively (within the mostly minus signature). For definiteness, we refer mostly to the $A d S$ case in this paper, although all formulae are valid also for the dS case.
} 
According to these definitions, $E^{A} V_{A} \equiv 0, D^{\mathcal{L}} V^{A}=d V^{A}+\omega^{\mathcal{L} A B} V_{B} \equiv 0$. The theory is formulated in a way independent of a particular choice of $V^{A}$. The simplest choice is with $V^{A}$ being a constant vector pointing at the $(d+1)^{t h}$ direction, i.e.,

$$
V^{A}=|\Lambda|^{-1 / 2} \delta_{d}^{A} .
$$

The Lorentz directions are those orthogonal to $V^{A}: V^{A} A_{A}=0 \rightarrow A_{A}=A_{a}$. In this "standard gauge" the Lorentz connection is $\omega^{a b}$, and $e^{a}=\omega^{a B} V_{B}$. When the frame $E_{n}^{A}$ has the maximal rank $d$, it gives rise to the nondegenerate metric tensor $g_{n m}=E_{n}^{A} E_{m}^{B} \eta_{A B}$.

The $o(d-1,2)$ Yang-Mills field strength is

$$
R^{A B}=d \omega^{A B}+\omega^{A} \wedge \omega^{C B} .
$$

It can be decomposed into the torsion part $R^{A} \equiv D E^{A} \equiv R^{A B} V_{B}$ and the $V$-transversal Lorentz part. In the standard gauge (2.3) they identify with the torsion tensor and Riemann tensor shifted by the terms bilinear in the frame 1 -form

$$
R^{a}=d e^{a}+\omega^{a}{ }_{b} \wedge e^{b}, \quad R^{a b}=d \omega^{a b}+\omega^{a}{ }_{c} \wedge \omega^{c b}+\Lambda e^{a} \wedge e^{b} .
$$

The zero-torsion condition $R^{a}=0$ expresses the Lorentz connection via the frame field in the usual manner. Provided that the metric tensor is nondegenerate, any field $\omega$ satisfying the zero-curvature equation $R^{A B}=0$ describes $(A) d S_{d}$ space with the cosmological constant $\Lambda$,

$$
A d S_{d}: \quad R^{A B}=0, \quad \operatorname{rank}\left|E_{n}^{A}\right|=d .
$$

The action of Stelle and West [24] for $4 d$ gravity is

$$
S=-\frac{1}{4 \kappa^{2}|\Lambda|^{1 / 2}} \int_{M^{4}} \varepsilon_{A B C D E} V^{A} R^{B C} R^{D E} .
$$

In the standard gauge it amounts to the action of MacDowell-Mansouri [23. To see that it is equivalent to the Einstein action with the cosmological term one decomposes $R^{a b}=$ $\mathcal{R}_{\mathcal{L}}^{a b}+\Lambda R_{\mathcal{C}}^{a b}$ where $R_{\mathcal{L}}^{a b}$ is the field strength of the Lorentz subalgebra $o(d-1,1)$ and $R_{\mathcal{C}}^{a b}=e^{a} \wedge e^{b}$. Plugging this into (2.7) one observes that the terms $R_{\mathcal{L}} \times R_{\mathcal{L}}$ form a topological invariant (Gauss-Bonnet), which does not contribute to the field equations, $R_{\mathcal{L}} \times R_{\mathcal{C}}$ terms form the scalar curvature and $R_{\mathcal{C}} \times R_{\mathcal{C}}$ terms give rise to the cosmological term.

The field $V^{A}$ makes the $o(d-1,2)$ gauge symmetry manifest

$$
\delta \omega^{A B}=D \epsilon^{A B}, \quad \delta V^{A}=-\epsilon^{A B} V_{B} .
$$

Also, the action (2.7) is manifestly invariant under diffeomorphisms because of using the exterior algebra formalism. Let us define a covariantized diffeomorphism transformation as a mixture of a usual diffeomorphism, generated by an infinitesimal vector field parameter $\xi^{n}$, with a local $o(d-1,2)$ gauge symmetry transformation with the parameter $\varepsilon_{\xi}^{A B}=$ $-\xi^{n} \omega_{n}^{A B}$. The transformation law under the covariantized diffeomorphisms is

$$
\delta_{\xi} V^{A}=\xi^{n} D_{n} V^{A} \equiv \xi^{n} E_{n}^{A},
$$




$$
\delta_{\xi} \omega_{m}^{A B}=\xi^{n} \partial_{n} \omega_{m}^{A B}+\partial_{m}\left(\xi^{n}\right) \omega_{n}^{A B}-D_{m}\left(\xi^{n} \omega_{n}^{A B}\right) \equiv \xi^{n} R_{n m}^{A B} .
$$

Fixing some gauge for $V^{A}$, one relates the parameters of diffeomorphisms and gauge transformations from $o(d-1,2) / o(d-1,1)$ via the condition

$$
\delta V^{A}=0=\xi^{n} E_{n}^{A}-\varepsilon^{A B} V_{B} .
$$

When $V^{A}$ is a constant vector, the condition does not restrict the parameters of true (i.e., noncovariantized) diffeomorphisms, rather restricting to zero the gauge parameters from $o(d-1,2) / o(d-1,1)$. The resulting theory turns out to be expressed in terms of the frame field and Lorentz connection contained in $\omega^{A B}$ and, as it should, is manifestly invariant under local Lorentz transformations and diffeomorphisms. Alternatively, expressing the covariantized diffeomorphism parameters from (2.11)

$$
\xi^{n} g_{n m}=E_{m A} \varepsilon^{A B} V_{B}
$$

and using (2.10), one can interpret the mixture of diffeomorphisms and transformations from $o(d-1,2) / o(d-1,1)$, that leave invariant $V^{A}$, as a deformation of the transformation law (2.8) for the connection $\omega^{A B}$ by some $R$-dependent terms.

With the help of $V_{A}$ it is straightforward to write a $d$-dimensional generalization [5] of the MacDowell-Mansouri-Stelle-West action

$$
S=-\frac{1}{4|\Lambda|^{1 / 2} \kappa^{d-2}} \int_{M^{d}} \epsilon_{A_{1} \ldots A_{d+1}} R^{A_{1} A_{2}} \wedge R^{A_{3} A_{4}} \wedge E^{A_{5}} \wedge \ldots \wedge E^{A_{d}} V^{A_{d+1}} .
$$

Obviously, among various solutions of the equations of motion of the action (2.13), any flat connection with $R^{A B}=0$ is a solution. According to (2.6) it describes $A d S_{d}$ provided that the metric is nondegenerate. (Different flat connections with nondegenerate metric describe $A d S_{d}$ in different coordinates.) This solution is most symmetric. Indeed, let $\omega_{0}^{A B}(x)$ be some solution of (2.6). According to (2.10), covariantized diffeomorphisms act trivially on $\omega_{0}$. Local $o(d-1,2)$ transformations (2.8) map one solution $\omega_{0}$ of (2.6) to another. The transformations, that leave $\omega_{0}$ invariant, satisfy

$$
D_{0} \varepsilon^{A B}(x)=0
$$

where $D_{0}$ is the $o(d-1,2)$ covariant derivative constructed from $\omega_{0}$. Equation (2.14) is formally consistent because $D_{0}^{2}=0$. As a result, it determines all derivatives of the 0-form $\varepsilon^{A B}(x)$ in terms of its values $\varepsilon^{A B}\left(x_{0}\right)$ at any given point $x_{0}$. So, in the topologically trivial situation, any solution $\varepsilon_{0}^{A B}(x)$ of (2.14) is fixed in terms of $\varepsilon_{0}^{A B}\left(x_{0}\right) \in o(d-1,2)$ which are arbitrary parameters of the global symmetry $o(d-1,2)$ of the vacuum (2.6). It is important to note that the same symmetry can be realized by diffeomorphisms generated by the Killing vector fields $\xi^{n}(2.12)$ of the metric $g_{0 n m}=E_{0 n}^{A} E_{0 m A}$ where $E_{0}$ is defined by (2.2) with the $A d S$ background connection $\omega_{0}{ }^{A B}$. This explains why the space-time symmetry algebras associated with the motions of the most symmetric vacuum spaces reappear as gauge symmetry algebras in the gauge approach to gravity.

The lesson is that, to describe a gauge model that has a global symmetry $h$, it is useful to reformulate it in terms of the gauge connections $\omega$ and curvatures $R$ of $h$ in such a way 
that the zero curvature equation $R=0$ solves the field equations. If a symmetry $h$ is not known, this observation can be used other way around to guess what it is by reformulating dynamics a la MacDowell-Mansouri to guess a structure of an appropriate curvature $R$.

\section{Higher spin gauge fields}

In the spin two case, the formulation in terms of gauge connections results from the extension $g_{n m} \longrightarrow\left\{e_{n}^{a}, \omega_{n}^{a b}\right\} \longrightarrow \omega_{n}^{A B}$. It has the following generalization to any spin

$$
s \geq 1: \quad \varphi_{n_{1} \ldots n_{s}} \longrightarrow\left\{e_{n}^{a_{1} \ldots a_{s-1}},\left.\omega_{n}^{a_{1} \ldots a_{s-1}, b_{1} \ldots b_{t}}\right|_{t=1,2 \ldots s-1}\right\} \longrightarrow \omega_{n}^{A_{1} \ldots A_{s-1}, B_{1} \ldots B_{s-1}}
$$

The first arrow is for the equivalent free field reformulation 1 t of the Fronsdal dynamics in terms of the set of 1 -forms $d x^{n} \omega_{n}{ }^{a_{1} \ldots a_{s-1}, b_{1} \ldots b_{t}}(0 \leq t \leq s-1)$ which contain the frame type dynamical field $e_{n}{ }^{a_{1} \ldots a_{s-1}}=\omega_{n}{ }^{a_{1} \ldots a_{s-1}}(t=0)$ and the generalized Lorentz connections $\omega^{a_{1} \ldots a_{s-1}, b_{1} \ldots b_{t}}(t>0)$. The connections $\omega^{a_{1} \ldots a_{s-1}, b_{1} \ldots b_{t}}$ are symmetric in the fiber Lorentz vector indices $a_{i}$ and $b_{j}$ separately, satisfy the antisymmetry condition

$$
\omega_{n}^{a_{1} \ldots a_{s-1}, a_{s} b_{2} \ldots b_{t}}=0
$$

implying that symmetrization over any $s$ fiber indices gives zero, and are traceless with

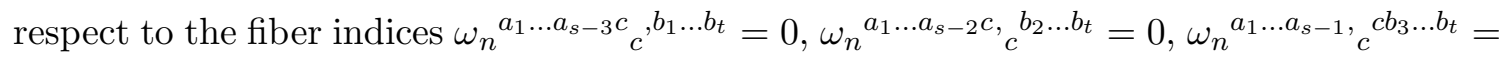
0 . The HS gauge fields associated with the spin $s$ massless field therefore take values in the direct sum of all irreducible representations of the $d$-dimensional massless Lorentz group $o(d-1,1)$ described by the_Young diagrams with at most two rows such that the longest row has length $s-1$. Analogously to the relationship between metric and frame formulations of the linearized gravity, the totally symmetric double traceless HS fields used to describe the HS dynamics in the metric type formalism 1 1 2 identify with the symmetrized part $\varphi_{a_{1} \ldots a_{s}}=\omega_{\left\{a_{1} \ldots a_{s}\right\}}$ of the frame type field $\omega_{n}{ }^{a_{1} \ldots a_{s-1}}$. The antisymmetric part in $\omega_{n}{ }^{a_{1} \ldots a_{s-1}}$ can be gauge fixed to zero with the aid of the generalized HS Lorentz symmetries with the parameter $\epsilon^{a_{1} \ldots a_{s-1}, b}$. That $\varphi_{a_{1} \ldots a_{s}}$ is double traceless is a consequence of the tracelessness of $\omega_{n}{ }^{a_{1} \ldots a_{s-1}}$ in the indices $a_{i}$. The generalized Lorentz connections $\omega_{n}{ }^{a(s-1), b(t)}$ with $t>0$ are auxiliary fields expressed through order- $t$ derivatives of the dynamical frame-like field by certain constrains, $\omega_{n}{ }^{a(s-1), b(t)} \sim\left(\frac{1}{\sqrt{\Lambda}} \frac{\partial}{\partial x}\right)^{t}(e)$.

The second arrow in (3.1) expresses the observation of [5] that the set of the HS 1-forms $d x^{n} \omega_{n}{ }^{a_{1} \ldots a_{s-1}, b_{1} \ldots b_{t}}$ with all $0 \leq t \leq s-1$ results from the "dimensional reduction" of a 1-form $d x^{n} \omega_{n} A_{1} \ldots A_{s-1}, B_{1} \ldots B_{s-1}$ carrying the irreducible representation of the $A d S_{d}$ algebra $o(d-1,2)$ described by the traceless two-row rectangular Young tableau of length $s-1$, i.e.

$$
\omega^{\left\{A_{1} \ldots A_{s-1}, A_{s}\right\} B_{2} \ldots B_{s-1}}=0, \quad \omega^{A_{1} \ldots A_{s-3} C}{ }_{C},{ }^{B_{1} \ldots B_{s-1}}=0 .
$$

The Lorentz covariant irreducible fields $d x^{n} \omega_{n}{ }^{a_{1} \ldots a_{s-1}, b_{1} \ldots b_{t}}$ identify with those components of $d x^{n} \omega_{n}{ }^{A_{1} \ldots A_{s-1}, B_{1} \ldots B_{s-1}}$ which are parallel to the compensator field $V^{A}$ in $s-t-1$ indices and are transversal in the rest ones. 
The linearized HS curvature $R_{1}$ has the form of the covariant derivative $D_{0}$ with the $o(d-1,2)$ connection $\omega_{0}^{A B}$ in the representation of $o(d-1,2)$ described by the two-row rectangular Young tableau of length $s-1$, i.e.

$$
\begin{gathered}
R_{1}^{A_{1} \ldots A_{s-1}, B_{1} \ldots B_{s-1}}=D_{0}\left(\omega^{A_{1} \ldots A_{s-1}, B_{1} \ldots B_{s-1}}\right)=d \omega^{A_{1} \ldots A_{s-1}, B_{1} \ldots B_{s-1}} \\
+(s-1)\left(\omega_{0}^{\left\{A_{1}\right.} C_{C} \wedge \omega^{\left.C A_{2} \ldots A_{s-1}\right\}, B_{1} \ldots B_{s-1}}+\omega_{0}^{\left\{B_{1}\right.} C \wedge \omega^{\left.A_{1} \ldots A_{s-1}, C B_{2} \ldots B_{s-1}\right\}}\right),
\end{gathered}
$$

where $\omega_{0}^{A B}$ is the background $A d S_{d}$ gauge field satisfying the flatness condition $D_{0}^{2}=0$ (2.6) which guarantees that the linearized curvature (3.4) is invariant under the Abelian HS gauge transformations with the HS gauge parameters $\epsilon^{A_{1} \ldots A_{s-1}, B_{1} \ldots B_{s-1}}$

$$
\delta \omega_{1}{ }^{A_{1} \ldots A_{s-1}, B_{1} \ldots B_{s-1}}=D_{0} \epsilon^{A_{1} \ldots A_{s-1}, B_{1} \ldots B_{s-1}} .
$$

The $o(d-1,2)$ covariant form of the free action for a massless spin $s$ field is [5]

$$
\begin{gathered}
S_{2}^{s}=\frac{1}{2} \sum_{p=0}^{s-2} a(s, p) \epsilon_{A_{1} \ldots A_{d+1}} \int_{M^{d}} E^{A_{5}} \wedge \ldots \wedge E^{A_{d}} V^{A_{d+1}} V_{C_{1}} \ldots V_{C_{2(s-2-p)}} \\
\wedge R_{1}^{A_{1} B_{1} \ldots B_{s-2}, A_{2} C_{1} \ldots C_{s-2-p} D_{1} \ldots D_{p}} \wedge R_{1}^{A_{3}{ }_{B} \ldots B_{s-2},{ }^{A_{4} C_{s-1-p} \ldots C_{2(s-2-p)} D_{1} \ldots D_{p}},}
\end{gathered}
$$

where

$$
a(s, p)=\tilde{a}(s)(-\Lambda)^{-(s-p-1)} \frac{(d-5+2(s-p-2)) ! !(s-p-1)}{(s-p-2) !} .
$$

The coefficients $a(s, p)$ are fixed up to an overall spin-dependent factor $\tilde{a}(s)$ by the "extra field decoupling condition" that the variation of the free action (B.6) is different from zero only for the fields $\omega_{n A_{1} \ldots A_{s-1}, B_{1}}=\omega_{n A_{1} \ldots A_{s-1}, B_{1} \ldots B_{s-1}} V^{B_{2}} \ldots V^{B_{s-1}}$ which contain the frame type dynamical HS field $\omega_{n}{ }^{a_{1} \ldots a_{s-1}}$ and the Lorentz type auxiliary field $\omega_{n}{ }^{a_{1} \ldots a_{s-1}, b}$, which is expressed in terms of the frame type field by virtue of its equation of motion equivalent to the "zero torsion condition" $R_{1} A_{1} \ldots A_{s-1}, B_{1} \ldots B_{s-1} V^{B_{1}} \ldots V^{B_{s-1}}=0$. Insertion of the expression for $\omega_{n}{ }^{a_{1} \ldots a_{s-1}, b}$ into (3.6) gives rise to the HS action expressed entirely (modulo total derivatives) in terms of $\omega_{n}^{a_{1} \ldots a_{s-1}}$ and its first derivatives. Since the linearized curvature (3.4) is invariant under the Abelian HS gauge transformations (3.5) the resulting action has necessary HS gauge symmetries and, because of the extra field decoupling condition, describes correctly the free field HS dynamics in $A d S_{d}$. In particular, the generalized Lorentz-like transformations with the gauge parameter $\epsilon_{A_{1} \ldots A_{s-1}, B_{1}}(x)$ guarantee that only the totally symmetric Fronsdal part $\varphi_{a_{1} \ldots a_{s}}=\omega_{\left\{a_{1} \ldots a_{s}\right\}}$ of the frame type gauge field contributes to the action.

\section{Higher spin algebras}

Once dynamics of totally symmetric HS gauge fields is shown to be described by 1-forms taking values in the two-row rectangular Young tableaux of $o(d-1,2)$, this suggests that a $A d S_{d}$ HS algebra $h$ admits a basis formed by a set of elements $T_{A_{1} \ldots A_{n}, B_{1} \ldots B_{n}}$, which satisfy the properties analogous to (3.3), $T_{\left\{A_{1} \ldots A_{s-1}, A_{s}\right\} B_{2} \ldots B_{s-1}}=0, T_{A_{1} \ldots A_{s-3} C C},{ }^{B_{1} \ldots B_{s-1}}=0$, and contains the $o(d-1,2)$ basis elements $T_{A, B}=-T_{B, A}$ such that

$$
\left[T_{C, D}, T_{A_{1} \ldots A_{s-1}, B_{1} \ldots B_{s-1}}\right]=\eta_{D A_{1}} T_{C A_{2} \ldots A_{s-1}, B_{1} \ldots B_{s-1}}+\ldots
$$


The question is whether there exists a non-Abelian algebra $h$ with these properties. If yes, the Abelian curvatures $R_{1}$ (3.4) can be understood as resulting from the linearization of the non-Abelian field curvatures $R$ of $h$ with the $h$ gauge connection $\tilde{\omega}=\omega_{0}+\omega$, where $\omega_{0}$ is some fixed flat zero-order connection of the $A d S_{d}$ subalgebra $o(d-1,2) \subset h$ and $\omega$ is the first-order dynamical part which describes massless fields of various spins. According to the discussion of section 2, any $h$ of this class is a candidate for a global HS algebra of the symmetric vacuum of a HS theory. The existence of such an algebra was indicated by the results of 25] where conserved currents (and therefore charges) in the free massless scalar field theory were shown to be described by various traceless two-row rectangular Young tableaux of the conformal algebra. A formal definition of $h$ as a conformal HS algebra of symmetries of a scalar field theory in $d-1$ dimension was given by Eastwood in [26. In this paper we use a slightly different definition of $h$ which is more suitable for the analysis of the HS interactions.

Consider oscillators $Y_{i}^{A}$ with $i=1,2$ satisfying the commutation relations

$$
\left[Y_{i}^{A}, Y_{j}^{B}\right]_{*}=\varepsilon_{i j} \eta^{A B}, \quad \varepsilon_{i j}=-\varepsilon_{j i}, \quad \varepsilon_{12}=1
$$

where $\eta^{A B}$ is the invariant metric of $o(d-1,2)$. (These oscillators can be interpreted as conjugated coordinates and momenta $Y_{1}^{A}=P^{A}, Y_{2}^{B}=Y^{B}$.) $\eta_{A B}$ and $\varepsilon^{i j}$ are used to raise and lower indices in the usual manner $A^{A}=\eta^{A B} A_{B}, a^{i}=\varepsilon^{i j} a_{j}, a_{i}=a^{j} \varepsilon_{j i}$.

We use the Weyl (Moyal) star product

$$
(f * g)(Y)=\frac{1}{\pi^{2(d+1)}} \int d S d T f(Y+S) g(Y+T) \exp -2 S_{i}^{A} T_{A}^{i} .
$$

$[f, g]_{*}=f * g-g * f,\{f, g\}_{*}=f * g+g * f$. The associative algebra of polynomials with the $*$ product law generated via (4.2) is called Weyl algebra $A_{d+1}$. Its generic element is $f(Y)=\sum \phi_{A_{1} \ldots A_{n}}^{i_{1} \ldots i_{n}} Y_{i_{1}}^{A_{1}} \ldots Y_{i_{n}}^{A_{n}}$ or, equivalently,

$$
f(Y)=\sum_{m, n} f_{A_{1} \ldots A_{m}, B_{1} \ldots B_{n}} Y_{1}^{A_{1}} \ldots Y_{1}^{A_{m}} Y_{2}^{B_{1}} \ldots Y_{2}^{B_{n}}
$$

with the coefficients $f_{A_{1} \ldots A_{m}, B_{1} \ldots B_{n}}$ symmetric in the indices $A_{i}$ and $B_{j}$.

Various bilinears of oscillators $Y_{i}^{A}$ form the Lie algebra $s p(2(d+1))$ with respect to the star commutator. It contains the subalgebra $o(d-1,2) \oplus s p(2)$ spanned by the mutually commuting generators

$$
T^{A, B}=-T^{B, A}=\frac{1}{2} Y^{i A} Y_{i}^{B}, \quad t_{i j}=t_{j i}=Y_{i}^{A} Y_{j A} \quad\left[T^{A, B}, t_{i j}\right]_{*}=0 .
$$

Consider the subalgebra $S \in A_{d+1}$ spanned by the $s p(2)$ singlets $f(Y)$

$$
f \in S: \quad\left[t_{i j}, f(Y)\right]_{*}=0 .
$$

Eq.(4.6) is equivalent to $\left(Y^{A i} \frac{\partial}{Y_{j}^{A}}+Y^{A j} \frac{\partial}{Y_{i}^{A}}\right) f(Y)=0$. For the expansion (4.4) this condition implies that the coefficients $f_{A_{1} \ldots A_{m}, B_{1} \ldots B_{n}}$ are nonzero only if $n=m$ and that symmetrization over any $m+1$ indices of $f_{A_{1} \ldots A_{m}, B_{1} \ldots B_{m}}$ gives zero, i.e. $f_{A_{1} \ldots A_{m}, B_{1} \ldots B_{m}}$ 
has the symmetry properties of a two-row rectangular Young tableau. The algebra $S$ is not simple. It contains the two-sided ideal $I$ spanned by the elements of the form $g=t_{i j} * g^{i j}$, where $g^{i j}$ transforms as a symmetric tensor with respect to $s p(2)$, i.e., $\left[t_{i j}, g^{k l}\right]_{*}=\delta_{i}^{k} g_{j}{ }^{l}+\delta_{j}^{k} g_{i}^{l}+\delta_{i}^{l} g_{j}{ }^{k}+\delta_{j}^{l} g_{i}{ }^{k}$. (Note that $t_{i j} * g^{i j}=g^{i j} * t_{i j}$.) Actually, from (4.6) it follows that $f * g, g * f \in I \forall f \in S, g \in I$. Due to the definition (4.5) of $t_{i j}$, the ideal $I$ contains all traces of the two-row Young tableaux. As a result, the algebra $S / I$ has only traceless two-row tableaux in the expansion (4.4).

Now consider the Lie algebra with the commutator in $S / I$ as the product law. Its real form corresponding to a unitary HS theory in $A d S_{d}$ is called $h u(1 / \operatorname{sp}(2)[d-1,2])$ [22]. Note that, by construction, the $A d S_{d}$ algebra $o(d-1,2)$ with the generators $T^{A, B}$ is the subalgebra of $h u(1 / s p(2)[n, m])$.

The gauge fields of $h u(1 / s p(2)[d-1,2])$ are

$$
\omega(Y \mid x)=\sum_{l=0}^{\infty} \omega_{A_{1} \ldots A_{l}, B_{1} \ldots B_{l}}(x) Y_{1}^{A_{1}} \ldots Y_{1}^{A_{l}} Y_{2}^{B_{1}} \ldots Y_{2}^{B_{l}}
$$

with the component gauge fields $\omega_{A_{1} \ldots A_{l}, B_{1} \ldots B_{l}}(x)$ taking values in all traceless two-row rectangular Young tableaux of $o(d-1,2)$. Note that, because $d t_{i j}=0$, the $s p(2)$ invariance condition, which imposes the Young symmetry properties, can be written in the covariant form

$$
D\left(t_{i j}\right) \equiv d t_{i j}+\left[\omega, t_{i j}\right]_{*}=0 .
$$

The HS curvatures and gauge transformations have the standard Yang-Mills form

$$
\begin{gathered}
R(Y \mid x)=d \omega(Y \mid x)+\omega(Y \mid x) \wedge * \omega(Y \mid x), \\
\delta \omega(Y \mid x)=D \varepsilon(Y \mid x), \quad D \varepsilon(Y \mid x)=d \varepsilon(Y \mid x)+[\omega(Y \mid x), \varepsilon(Y \mid x)]_{*} .
\end{gathered}
$$

Different spins correspond to irreducible representations of $o(d-1,2)$ spanned by homogeneous polynomials

$$
\omega(\mu Y \mid x)=\mu^{2(s-1)} \omega(Y \mid x) .
$$

(Note that one unit of spin is carried by the 1 -form index). In particular, spin 1 is described by a 1 -form $\omega(x)=d x^{n} \omega_{n}(x)$. The algebra $h u(1 / s p(2)[d-1,2])$ is infinite dimensional. It contains $o(d-1,2) \oplus u(1)$ as the maximal finite dimensional subalgebra with the generators $T^{A B}$ (4.5) for $o(d-1,2)$ and constants for $u(1)$. The corresponding gauge fields carry spins 2 and 1 , respectively. Taking two HS symmetry parameters $\varepsilon_{s_{1}}$ and $\varepsilon_{s_{2}}$, being polynomials of degrees $s_{1}-1$ and $s_{2}-1$, respectively, one obtains

$$
\left[\varepsilon_{s_{1}}, \varepsilon_{s_{2}}\right]_{*}=\sum_{t=\left|s_{1}-s_{2}\right|+1}^{s_{1}+s_{2}-2} \varepsilon_{t} .
$$

Thus, once a spin $s>2$ gauge field appears, the HS symmetry algebra requires an infinite tower of HS gauge fields to be present. The barrier $s \leq 2$ separates theories with infinite dimensional gauge symmetries from those with usual lower spin symmetries. It is tempting to speculate that the latter result from the spontaneous breaking of infinite dimensional 
HS symmetries down to usual lower spin symmetries. In that case, the HS gauge fields should acquire masses as a result of this spontaneous HS symmetry breaking.

The formula (4.12) manifests the quantum-mechanical nonlocality of the oscillator algebra (4.2) (equivalently, the star product (4.3)). Because bilinear terms in the HS curvatures (4.9) describe interactions, one concludes that lower spins form sources for higher spins and vice versa. A less obvious fact, which follows from the HS field equations, is that the nonlocal character of the star product algebra results in the appearance of higher space-time derivatives in the HS interactions. Thus the star-product origin of the HS algebra links together such seemingly different properties of the HS theories as the relevance of the $A d S$ background, necessity of introducing infinitely many spins and spacetime non-locality of the HS interactions. Note that these properties make the HS theories reminiscent of the superstring theory with the analogy between the cosmological constant and $\alpha^{\prime}$.

To introduce inner symmetries, one considers following [27] matrix-valued gauge fields $\omega(Y \mid x) \longrightarrow \omega_{\nu}^{\mu}(Y \mid x), \mu, \nu \ldots=1, \ldots, p$, imposing the reality condition

$$
\left[\omega_{\nu}^{\mu}(Y \mid x)\right]^{\dagger}=-\omega_{\nu}^{\mu}(Y \mid x),
$$

where the involution $\dagger$ combines matrix hermitian conjugation with the involution of the star product algebra $\left(Y_{j}^{A}\right)^{\dagger}=i Y_{j}^{A}$. The resulting real Lie algebra is called $h u(p|s p(2)|[d-$ $1,2])$. Its gauge fields describe the set of massless fields of all spins $s \geq 1$ which take values in the adjoint representation of $u(p)$. In particular, spin 1 gauge fields are $u(p)$ Yang-Mills fields.

Combining the antiautomorphism of the star product algebra $\rho(f(Y))=f(i Y)$ with some antiautomorphism of the matrix algebra generated by a nondegenerate form $\rho_{\alpha \beta}$ one can impose the conditions [27]

$$
\omega_{\alpha}^{\beta}(Y \mid x)=-\rho^{\beta \gamma} \rho_{\delta \alpha} \omega_{\gamma}^{\delta}(i Y \mid x),
$$

which truncate the original system to the one with the Yang-Mills gauge group $\operatorname{USp}(p)$ or $O(p)$ depending on whether the form $\rho_{\alpha \beta}$ is antisymmetric or symmetric, respectively. The corresponding global HS symmetry algebras are called $h u s p(p \mid s p(2)[d-1,2])$ and $h o(p \mid s p(2)[d-1,2])$, respectively. In this case all fields of odd spins take values in the adjoint representation of the Yang-Mills group while fields of even spins take values in the opposite symmetry second rank tensor representation (i.e., symmetric for $O(p)$ and antisymmetric for $U S p(p))$ which contains a singlet. The graviton is always the color singlet. For general $p$, color spin 2 particles also appear however. Note that this does not contradict to the no-go results of [28] because the theory under consideration does not allow a flat limit with unbroken HS and color spin 2 symmetries. The minimal HS theory is based on the algebra $h o(1 \mid s p(2)[n, m])$. It describes even spin particles, each in one copy. (Odd spins do not appear because the adjoint representation of $o(1)$ is trivial.)

\section{Unfolded higher spin dynamics}

An efficient approach to HS dynamics consists of reformulation of linear and non-linear field equations in the form of some generalized covariant constancy conditions first at the 
linear and then at the nonlinear level. This "unfolded formulation" originally introduced for the description of $4 d$ HS dynamics in [29] allows one to control simultaneously formal consistency of field equations, gauge symmetries and the invariance under diffeomorphisms. The unfolded formulation treats uniformly higher derivatives of the dynamical fields and is just appropriate for the analysis of HS dynamics because HS symmetries mix higher derivatives of the dynamical fields. The same time, the unfolded formulation is a universal tool applicable to any dynamical system although it may be looking unusual from the perspective of standard field theory because it operates in terms of infinite dimensional modules which describe all degrees of freedom of the system in question. To illustrate the idea let us recall the unfolded formulation of the Einstein gravity, using the compensator formalism.

As explained in section 2, Riemann tensor and torsion tensor are components of the $o(d-1,2)$ field strength (2.4) $R^{A B}=d x^{n} \wedge d x^{m} R_{n m}{ }^{A B}$. The components of the Riemann tensor, that can be nonzero when Einstein equations and zero-torsion constraints are satisfied, belong to the Weyl tensor, i.e. Einstein equations with the cosmological term can be rewritten as

$$
\left.R^{A, B}\right|_{\text {o.m.s. }}=E_{C} \wedge E_{D} C^{A C, B D}
$$

where $C^{A C, B D}$ is treated as an independent tensor field variable that has the symmetry properties of the window Young tableau $\boxplus$ and describes the Weyl tensor. For our purpose it is convenient to use the symmetric basis with $C^{A C, B D}=C^{C A, B D}=C^{A C, D B}$. In addition, $C^{A C, B D}$ has the following properties

$$
\begin{aligned}
\text { zero-torsion constraint : } & V_{A} C^{A B, C D}=0, \\
\text { Einstein equations : } & C_{B}{ }^{B, C D}=0, \\
\text { Bianchi identities for (5.2) : } & C^{\left\{A_{1} A_{2}, A_{3}\right\} D}=0: \quad \text { 田. }
\end{aligned}
$$

Field equations for free totally symmetric integer spin $s \geq 2$ massless HS fields in $A d S_{d}$ [4] can analogously be rewritten in the form [4]

$$
\left.R_{1}^{A_{1} \ldots A_{s-1}, B_{1} \ldots B_{s-1}}\right|_{\text {o.m.s. }}=E_{0 A_{s}} \wedge E_{0 B_{s}} C^{A_{1} \ldots A_{s}, B_{1} \ldots B_{s}} .
$$

The generalized Weyl tensors $C^{A_{1} \ldots A_{s}, B_{1} \ldots B_{s}}$ are described by the traceless $V^{A_{-} \text {transversal }}$ two-row rectangular Young tableaux of length $s$, i.e., $V_{A_{1}} C^{A_{1} \ldots A_{s}, B_{1} \ldots B_{s}}=0, \eta_{A_{1} A_{2}} C^{A_{1} \ldots A_{s}, B_{1} \ldots B_{s}}=$ 0 and $C^{\left\{A_{1} \ldots A_{s}, A_{s+1}\right\} B_{2} \ldots B_{s}}=0$. The equation (5.5) referred to as First On-Mass-Shell Theorem is a consequence of the massless field equations along with the constraints on auxiliary and extra fields imposed by requiring appropriate components of HS curvature to vanish [4]. Let us note that although the extra fields $\omega_{n}{ }^{a_{1} \ldots a_{s-1}, b_{1} \ldots b_{t}}$ with $t \geq 2$ do not contribute to the free action, they do contribute at the interaction level. To make such interactions meaningful, one has to express the extra fields in terms of the dynamical ones modulo pure gauge degrees of freedom. This is achieved by imposing appropriate constraints [4 contained in (5.5) like the torsion constraint in gravity is contained in (5.1).

The Bianchi identities $D_{0}\left(R_{1}\right)=0$ along with the equation (5.5) impose some differential restrictions on the generalized Weyl tensor $C^{A_{1} \ldots A_{s}, B_{1} \ldots B_{s}}$. The trick is to denote the 
components of the first derivatives of $C^{A_{1} \ldots A_{s}, B_{1} \ldots B_{s}}$, that are allowed to be non-zero by the Bianchi identities, by a new tensor field $C_{1}$, writing symbolically $D_{0}^{\mathcal{L} C}=E_{0} \wedge C_{1}$, where $D_{0}^{\mathcal{L}}$ is the Lorentz derivative and $E_{0}$ is the $A d S$ frame 1 -form. The Bianchi identities for this equation impose differential conditions on $C_{1}$ to be written as $D_{0}^{\mathcal{L}} C_{1}=E_{0} \wedge C_{2}$, etc. It turns out that the full set of the 0 -forms $C_{i}$ consists of all two-row traceless $V^{A}$-transversal Young tableaux $C^{A_{1} \ldots A_{u}, B_{1} \ldots B_{s}}$ with the second row of length $s$, i.e.,

$$
V_{A_{1}} C^{A_{1} \ldots A_{u}, B_{1} \ldots B_{s}}=0, \quad \eta_{A_{1} A_{2}} C^{A_{1} \ldots A_{u}, B_{1} \ldots B_{s}}=0, \quad C^{\left\{A_{1} \ldots A_{u}, A_{u+1}\right\} B_{2} \ldots B_{s}}=0 .
$$

The fields $C^{A_{1} \ldots A_{u}, B_{1} \ldots B_{s}}$ form a basis of the space of on-mass-shell nontrivial derivatives of order $u-s$ of the spin $s$ generalized Weyl tensor $C^{A_{1} \ldots A_{s}, B_{1} \ldots B_{s}}$. The full set of the compatibility conditions of the equations (5.5) can be written in the form of the covariant constancy condition [22]

$$
\tilde{D}_{0} C^{A_{1} \ldots A_{u}, B_{1} \ldots B_{s}}=0 \quad u \geq s,
$$

where $\tilde{D}_{0}$ is the $o(d-1,2)$ covariant derivative in the so called twisted adjoint representation.

To define the twisted adjoint representation it is useful to observe that the set of fields $C^{A_{1} \ldots A_{u}, B_{1} \ldots B_{s}}$ satisfying (5.6) spans the space isomorphic to the space of star-product functions $C(Y \mid x)$ satisfying (4.6) and with the ideal $I$ factored out to impose the tracelessness condition. Then $\tilde{D}_{0}$ is 22

$$
\tilde{D}_{0}=D_{0}^{\mathcal{L}}-2 \Lambda E_{0}^{A} V^{B}\left({ }^{\perp} Y_{A}^{i \|} Y_{B i}-\frac{1}{4} \varepsilon^{j i} \frac{\partial}{\partial^{\perp} Y^{A j} \partial^{\|} Y^{B i}}\right),
$$

where the Lorentz covariant derivative is $D_{0}^{\mathcal{L}}=d+\omega_{0}^{\mathcal{L}}{ }^{A B \perp} Y_{A i} \frac{\partial}{\partial^{\perp} Y_{i}^{B}}$ and we use notations

$$
A_{i}^{A}={ }^{\|} A_{i}^{A}+{ }^{\perp} A_{i}^{A}, \quad{ }^{\|} A_{i}^{A}=\frac{1}{V^{2}} V^{A} V_{B} A_{i}^{B}, \quad{ }^{\perp} A_{i}^{A}=A_{i}^{A}-\frac{1}{V^{2}} V^{A} V_{B} A_{i}^{B}
$$

for any vector $A^{A}$. The twisted adjoint covariant derivative (5.8) commutes with the operator $N^{t w}={ }^{\perp} Y_{i}^{A} \frac{\partial}{\partial^{\perp} Y_{i}^{A}}-{ }^{\|} Y_{i}^{A} \frac{\partial}{\partial \| Y_{i}^{A}}$. This means that the equation (5.7) decomposes into independent subsystems for the sets of fields satisfying $N^{t w} C=2 s C$ with various integers $s \geq 0$ (the eigenvalues of the operator $N^{t w}$ are non-negative on the space of $o(d-1,2)$ tensors with the symmetry property of a two-row rectangular Young tableau because having more than a half of vector indices aligned along $V^{A}$ would imply symmetrization over more than a half of indices, thus giving zero). Different Lorentz irreducible components of the field $C(Y \mid x)$ with some fixed $s$ just give the set of components $C^{A_{1} \ldots A_{u}, B_{1} \ldots B_{s}}$ satisfying (5.6) with $u-s$ being a number of indices of the length $u$ two-row traceless rectangular tableau, contracted with the compensator $V^{A}$. Note that any spin $s$ submodule of the twisted adjoint $o(d-1,2)$ module, where the 0 -forms $C$ take their values, is infinite dimensional. This is expected because the components $C$ parametrize all gauge invariant combinations of on-mass-shell non-zero derivatives of the higher spin fields.

Equations (5.5) and (5.7) were originally derived [4, 5] by the equivalent reformulation (unfolding) of the free equations of motion of totally symmetric massless fields of all integer 
spins $s \geq 2$ in $A d S_{d}$ supplemented with some constraints which express an infinite set of auxiliary variables via higher derivatives of the dynamical fields. However, the equation of motion of a massless scalar coincide [30] with the $s=0$ sector of equation (5.7). Analogously, equation (5.7) with $s=1$ imposes the Maxwell equations on the spin 1 potential (1-form) $\omega$ on the left hand side of (5.5).

Thus, the equations (5.5) and (5.7) give an equivalent form of bosonic symmetric massless fields of all spins in $A d S_{d}$ for any $d$. We call this important fact Central OnMass-Shell Theorem. It is of key importance in many respects and, in particular, for the analysis of interactions. Central On-Mass-Shell theorem can be formulated in the following compact form

$$
\begin{gathered}
R_{1}\left({ }^{\|} Y,{ }^{\perp} Y \mid x\right)=\frac{1}{2} E_{0}^{A} \wedge E_{0}^{B} \frac{\partial^{2}}{\partial Y_{i}^{A} \partial Y_{j}^{B}} \varepsilon_{i j} C\left(0,{ }^{\perp} Y \mid x\right), \\
\tilde{D}_{0}(C)=0,
\end{gathered}
$$

where $R_{1}=d \omega+\omega_{0} * \omega+\omega * \omega_{0}, \tilde{D}_{0}(C)=d C+\omega_{0} * C-C * \tilde{\omega}_{0}$ and $\omega_{0}=\omega_{0}^{A B}(x) T_{A B}(Y)$ where $\omega_{0}^{A B}(x)$ satisfies (2.6) to describe the $(A) d S_{d}$ background, and tilde denotes the $V$ reflection automorphism, i.e., $\tilde{\omega}_{0}(Y \mid x)=\omega_{0}(\tilde{Y} \mid x)=\omega_{0}\left({ }^{\perp} Y-\| Y \mid x\right)$. The $V$-transversal components of the expansion of the 0 -forms $C\left(0,{ }^{\perp} Y\right)$ on the r.h.s. of (5.10) in powers of $Y_{i}^{A}$ give rise to HS Weyl 0-forms $C^{a_{1} \ldots a_{s}, b_{1} \ldots b_{s}}$ on the r.h.s. of (5.5). The key fact is that, as one can readily see, the equations (5.10) and (5.11) are consistent, i.e., the application of the covariant derivative to the l.h.s. of (5.10) and (5.11) does not lead to new conditions.

\section{Nonlinear higher spin classical dynamics}

The problem is to find a nonlinear deformation of the equations (5.10) and (5.11) in which the linearized curvature and covariant derivative are replaced with the full ones with $\omega=\omega_{0}+\omega_{1}$ where $\omega_{0}$ describes the background $A d S_{d}$ space-time and $\omega_{1}$ describes the dynamical HS gauge fields. According to the general analysis of [29], where the $4 d$ case was considered, any deformation, formulated using the exterior algebra formalism, that is consistent with the Bianchi identities, describes gauge invariant interactions and is invariant under diffeomorphisms. Here we formulate following [22] a system of equations which generates all nonlinear corrections to the equations (5.10) and (5.11). Before going into technical details let us stress that the solution we have found gives a deformation which is unique modulo field redefinitions. This means that all dimensionless coupling constants can be rescaled away in the classical HS model like the dimensionless Yang-Mills constant $g^{2}=|\Lambda|^{\frac{d-2}{2}} \kappa^{2}$ in the classical pure Yang-Mills theory. The only nontrivial ambiguity that remains is to consider HS theories with different HS algebras corresponding to different Yang-Mills groups according to the classification of section ta

Roughly speaking, the idea is to describe complicated nonlinear corrections to HS equations as a solution of some simple differential type equations with respect to additional variables. To this end we double a number of oscillators by introducing additional variables $Z_{i}^{A}$. The full system of equations is formulated in terms of the fields $W(Z, Y \mid x)$, $B(Z, Y \mid x)$ and $S(Z, Y \mid x)$, where $B(Z, Y \mid x)$ is a 0 -form while $W(Z, Y \mid x)=d x^{n} W_{n}(Z, Y \mid x)$ 
and $S(Z, Y \mid x)=d Z_{i}^{A} S_{A}^{i}(Z, Y \mid x)$ are connection 1-forms in space-time and auxiliary $Z_{i}^{A}$ directions, respectively. The fields $\omega$ and $C$ are identified with the "initial data" for the evolution in $Z$ variables as follows $\omega(Y \mid x)=W(0, Y \mid x), C(Y \mid x)=B(0, Y \mid x)$. The $Z$ - connection $S$ will be determined in terms of $B$ modulo gauge ambiguity. The differentials satisfy the standard anticommutation relations $d x^{n} d x^{m}=-d x^{m} d x^{n}, d Z_{i}^{A} d Z_{j}^{B}=-d Z_{j}^{B} d Z_{i}^{A}$, $d x^{n} d Z_{j}^{B}=-d Z_{j}^{B} d x^{n}$ and commute to all other variables (from now on we discard the wedge symbol).

The space of functions $f(Z, Y)$ is endowed with the star product

$$
(f * g)(Z, Y)=\frac{1}{\pi^{2(d+1)}} \int d S d T f(Z+S, Y+S) g(Z-T, Y+T) \exp -2 S_{i}^{A} T_{A}^{i},
$$

which is associative, normalized so that $1 * f=f * 1=f$ and gives rise to the commutation relations $\left[Y_{i}^{A}, Y_{j}^{B}\right]_{*}=\varepsilon_{i j} \eta^{A B},\left[Z_{i}^{A}, Z_{j}^{B}\right]_{*}=-\varepsilon_{i j} \eta^{A B},\left[Y_{i}^{A}, Z_{j}^{B}\right]_{*}=0$. The star product (6.1) describes a normal-ordered basis in $A_{2(n+m)}$ with respect to creation and annihilation operators $Z-Y$ and $Y+Z$, respectively.

Important property of the star product (6.1) is that it admits the inner Klein operator

$$
\mathcal{K}=\exp -2 z_{i} y^{i}, \quad y_{i}=\frac{1}{\sqrt{V^{2}}} V_{B} Y_{i}^{B}, \quad z_{i}=\frac{1}{\sqrt{V^{2}}} V_{B} Z_{i}^{B},
$$

which has the properties

$$
\mathcal{K} * f=\tilde{f} * \mathcal{K}, \quad \mathcal{K} * \mathcal{K}=1,
$$

where $\tilde{f}(Z, Y)=f(\tilde{Z}, \tilde{Y})$ with $\tilde{A}^{A}={ }^{\perp} A^{A}-{ }^{\|} A^{\perp}$ for $A^{A}=Z^{A}, Y^{A} \ldots$

The full nonlinear system of HS equations is [22]

$$
\begin{gathered}
d W+W * W=0, \quad d S+W * S+S * W=0, \quad d B+W * B-B * \tilde{W}=0, \\
S * S=-\frac{1}{2}\left(d Z_{i}^{A} d Z_{A}^{i}+4 \Lambda^{-1} d z_{i} d z^{i} B * \mathcal{K}\right), \quad S * B=B * \tilde{S},
\end{gathered}
$$

where $\tilde{S}(d Z, Z, Y)=S(\tilde{d Z}, \tilde{Z}, \tilde{Y})$ and $d z_{i}=\frac{1}{\sqrt{V^{2}}} V_{B} d Z_{i}^{B}$. In terms of a noncommutative connection $\mathcal{W}=d+W+S$ the system (6.4), (6.5) reads

$$
\mathcal{W} * \mathcal{W}=-\frac{1}{2}\left(d Z_{i}^{A} d Z_{A}^{i}+4 \Lambda^{-1} d z_{i} d z^{i} B * \mathcal{K}\right), \quad \mathcal{W} * B=B * \tilde{\mathcal{W}} .
$$

We see that $d z_{i} d z^{i} B * \mathcal{K}$ is the only nonzero component of the noncommutative curvature. The $B$-dependent part of the equation (6.5) is responsible for interactions. Note that $B$ has dimension $\mathrm{cm}^{-2}$ to match the Central On-Mass-Shell theorem (5.10) upon identification of $B$ with $C$ in the lowest order, i.e., $\Lambda^{-1} B$ is dimensionless. This form of the nonzero part of the non-commutative curvature manifests that taking the flat limit may be difficult in the interacting theory unless a value of the cosmological constant is shifted by a condensate which breaks the HS gauge symmetries.

The system is formally consistent in the sense that the associativity relations $\mathcal{W} *(\mathcal{W} *$ $\mathcal{W})=(\mathcal{W} * \mathcal{W}) * \mathcal{W}$ and $(\mathcal{W} * \mathcal{W}) * B=B *(\mathcal{W} * \mathcal{W})$, equivalent to Bianchi identities, are respected by the equations (6.4), (6.5). The only nontrivial part of the consistency check is that for the relationship $(S * S) * S=S *(S * S)$ in the sector of $\left(d z_{i}\right)^{3}$ due to 
the second term on the r.h.s. of (b.5) since $B * \mathcal{K}$ commutes to everything except for $d z_{i}$ to which it anticommutes by the second equation in (6.5). However, this does not break down the consistency of the system because $\left(d z_{i}\right)^{3} \equiv 0$. As a result, the equations (6.4), (6.5) are consistent as "differential" equations with respect to $x$ and $Z$ variables. A related statement is that the equations (6.4), (6.5) are invariant under the gauge transformations

$$
\delta \mathcal{W}=[\varepsilon, \mathcal{W}]_{*}, \quad \delta B=\varepsilon * B-B * \tilde{\varepsilon}
$$

with an arbitrary gauge parameter $\varepsilon(Z, Y \mid x)$.

To analyze the equations (6.4), (6.5) perturbatively one sets $W=W_{0}+W_{1}, S=S_{0}+S_{1}$ and $B=B_{0}+B_{1}$ with the vacuum solution $B_{0}=0, S_{0}=d Z_{i}^{A} Z_{A}^{i}$ and $W_{0}=\frac{1}{2} \omega_{0}^{A B}(x) Y_{A}^{i} Y_{i B}$, where $\omega_{0}^{A B}(x)$ satisfies the zero curvature conditions to describe $(A) d S_{d}$. As shown in [22], the nontrivial part of the system (6.4), (5.5) in the lowest order has the form (5.10), (5.11). The same time the system (6.4)-(6.5) generates all nonlinear corrections to the unfolded free HS equations (5.10), (5.11).

An intriguing feature of the unfolded formulation of the nonlinear HS equations is that their nontrivial part (6.5) has a form of deformed oscillator algebra equivalent [31] to a two-dimensional fuzzy hyperboloid in the "auxiliary" non-commutative space with the coordinates $Z, Y$. The origin of this fact can be traced back to the condition that the theory must admit unbroken $s p(2)$ symmetry with some nonlinearly deformed generators $t_{i j}$ satisfying the covariant constancy condition (4.8) at the nonlinear level to guarantee that the interacting HS theory admits interpretation in terms of the same set of tensor fields as the free theory (see [22] for more details). According to [31], from (6.6) it follows that the radius of the fuzzy hyperboloid is $R_{H^{2}}(x) \sim\left(4 \Lambda^{-1} B(x)+1\right) *\left(4 \Lambda^{-1} B(x)-3\right)$. Note that it depends via $B$ on a value of the HS curvature (including the gravitational one) at a given point $x$ of the space-time base manifold.

\section{Conclusion}

The main conclusion is that there exists a class of consistent nonlinear HS gauge theories in anti-de Sitter space of any dimension. These theories describe totally symmetric bosonic fields of all integer spins and are fixed uniquely by the HS gauge symmetry principle modulo the choice of the spin 1 Yang-Mills group which can be $U(n), O(n)$ or $S p(2 n)$. Global HS symmetries of the most symmetric vacua of HS gauge theories are certain star product algebras which exhibit usual quantum-mechanical nonlocality in the auxiliary noncommutative spaces. The field equations in the HS gauge theory map this nonlocality to space-time nonlocality (i.e., higher derivatives) at the interaction level. The same time the HS gauge theories remain local at the linearized level because the space-time symmetries are realized in terms of bilinears of auxiliary oscillators. Let us note that analogously to the non-commutative geometry framework of string theory, HS theory is based on the associative algebras (i.e., star product and its matrix extension). For this reason, in particular, there is no HS model with the spin 1 gauge group $S U(n)$.

Finally, let us list some open problems important for elucidating the structure of HS gauge theories in higher dimensions and their possible relationship with string theory. 
- To develop a theory of mixed symmetry (i.e. neither totally symmetric nor totally antisymmetric) HS gauge fields in $A d S_{d}$. This problem is not completely trivial in view of the observation of [32] that not every free mixed symmetry field in Minkowski space allows a smooth deformation to $A d S_{d}$. The origin of this phenomenon is [33] that some of the HS gauge symmetries of the flat space mixed symmetry fields turn out to be broken in the $A d S$ background. This means that the covariant description of generic massless fields in $A d S_{d}$ requires separate investigation compared to the flat space [15]. A progress in this direction achieved recently in [34, 35, 36 indicates that the general problem has a good chance to be solved soon. The formulation of [35] in terms of HS gauge potentials analogous to that used to describe symmetric HS fields looks particularly promising from the perspective of elucidating a structure of HS algebras underlying HS gauge theories with mixed symmetry fields.

- As a nontrivial consistency test, it is instructive to check whether the HS algebras admit unitary representations with the spectra of spins of massless fields equivalent to those predicted by the consistent nonlinear field equations (6.6).

- To extend the formulation of the nonlinear HS dynamics to the action level.

- To find solutions of HS field equations which break down HS gauge symmetries and introduce a massive parameter $m$ different from the cosmological constant. This is necessary to define a low energy expansion in $\left(\frac{1}{m} \frac{\partial}{\partial x}\right)^{p}$. Note that the cosmological constant $\Lambda$ cannot be used for this purpose because the dimensionless operator $\Lambda^{-\frac{1}{2}} D$ is of order one as a consequence of (1.3), i.e. an expansion in powers of derivatives in $A d S$ space without any other dimensionful parameter $m$ has formal meaning.

Acknowledgement This research was supported in part by grants INTAS No.03-516346, RFBR No.02-02-17067 and LSS No.1578.2003-2.

\section{References}

[1] C. Fronsdal, Phys. Rev. D18 (1978) 3624.

[2] B. de Wit and D.Z. Freedman, Phys. Rev. D21 (1980) 358.

[3] D. Francia and A. Sagnotti, Phys.Lett. B543 (2002) 303, hep-th/0207002; "On the geometry of higher spin gauge fields", hep-th/0212185.

[4] M. A. Vasiliev, Yad.Fiz. 32 (1980) 855;

V.E. Lopatin and M.A. Vasiliev, Mod. Phys. Lett. A3 (1988) 257.

[5] M.A. Vasiliev, Nucl. Phys. B616 (2001) 106, hep-th/0106200.

[6] P. van Nieuwenhuizen, Phys. Rep. 68 (1981) 189.

[7] E. Witten, Nucl. Phys. B268 (1986) 253.

[8] D. Gross, Phys. Rev. Lett. 60 (1988) 1229.

[9] E.S. Fradkin and M.A. Vasiliev, Phys. Lett. B189 (1987) 89; Nucl. Phys. B291 (1987) 141. 
[10] B. Sundborg, Nucl. Phys. Proc. Suppl. 102 (2001) 113, hep-th/0103247;

E. Witten, talk at J.H. Schwarz 60-th Birthday Conference, CalTech, November 2-3, 2001, http://theory. caltech.edu/jhs60/witten/1.html;

O.V. Shaynkman and M.A. Vasiliev, Theor. Math. Phys. 128 (2001) 1155, hep-th/0103208;

I. R. Klebanov and A. M. Polyakov, Phys. Lett. B550 (2002) 213, hep-th/0210114;

T. Leonhardt, A. Meziane and W. Ruhl, Phys. Lett. B555 (2003) 271, hep-th/0211092;

T. Leonhardt and W. Ruhl, "The minimal conformal $\mathrm{O}(\mathrm{N})$ vector sigma model at $\mathrm{d}=3$ ", hep-th/0308111;

L.Girardello, M.Porrati and A.Zaffaroni, Phys. Lett. B561 (2003) 289, hep-th/0212181;

A.C. Petkou, JHEP 0303 (2003) 049, hep-th/0302063;

R.G. Leigh and A.C. Petkou, JHEP 0306 (2003) 011, hep-th/0304217;

M. Bianchi, J.F. Morales and H. Samtleben, JHEP 0307 (2003) 062, hep-th/0305052;

U. Lindstrom and M. Zabzine, "Tensionless strings, WZW models at critical level and massless higher spin fields", hep-th/0305098;

R.Gopakumar, "From free fields to $A d S$ ", hep-th/0308184.

[11] E. Sezgin and P. Sundell, JHEP 0109:036, (2001), hep-th/0105001.

[12] M. A. Vasiliev, Phys. Rev. D66 (2002) 066006, hep-th/0106149.

[13] J. M. Maldacena, Adv. Theor. Math. Phys. 2 (1998) 231 [Int. J. Theor. Phys. 38 (1999) 1113], hep-th/9711200.

[14] R.R. Metsaev and A.A. Tseytlin, Phys.Rev. D63 (2001) 046002, hep-th/0007036;

J.Math.Phys. 42 (2001) 2987, hep-th/0011191.

[15] T. Curtright, Phys. Lett. B165 (1985) 304;

C. S. Aulakh, I. G. Koh and S. Ouvry, Phys. Lett. B173 (1986) 284;

W. Siegel and B. Zwiebach, Nucl. Phys. B282 (1987) 125;

J.M.F. Labastida, Phys. Rev. Lett. 58 (1987) 531; Nucl. Phys. B322 (1989) 185;

A. Pashnev and M. M. Tsulaia, Mod. Phys. Lett. A12 (1997) 861, hep-th/9703010; Mod.

Phys. Lett. A13 (1998) 1853, hep-th/9803207;

C. Burdik, A. Pashnev and M. Tsulaia, Mod. Phys. Lett. A16 (2001) 731, hep-th/0101201;

Nucl. Phys. Proc. Suppl 102 (2001) 285, hep-th/0103143;

$\mathrm{X}$. Bekaert and N. Boulanger, "Tensor gauge fields in arbitrary representations of GL(D,R): duality and Poincare lemma", hep-th/0208058; Phys. Lett. B561 (2003) 183,

hep-th/0301243;

P. de Medeiros and C. Hull,JHEP 0305 (2003) 019, hep-th/0303036.

[16] S. Deser and A. Waldron, Nucl. Phys B607 (2001) 577, hep-th/0103198.

[17] S. Coleman and J. Mandula, Phys. Rev. D159, 1251 (1967);

R. Haag, J. Lopuszanski and M. Sohnius, Nucl. Phys. B88 (1975) 257.

[18] C. Aragone and S. Deser, Phys. Lett. B86 (1979) 161.

[19] A.Bengtsson, I.Bengtsson and L.Brink, Nucl. Phys. B227 (1983) 31, 41;

A.Bengtsson and I.Bengtsson, Class. Quant. Grav. 3 (1986) 927;

A.Bengtsson, Phys. Lett. B182 (1986) 321; Class. Quant. Grav. 5 (1988) 437;

E.S. Fradkin and R.R. Metsaev, Class. Quant. Grav. 8 (1991) L89;

R.R. Metsaev, Mod. Phys. Lett. A6 (1991) 359, 2411; Mod. Phys. Lett. A8 (1993) 2413;

Class. Quant. Grav. 10 (1993) L39; Phys. Lett. B309 (1993) 39. 
[20] F.A. Berends, G.J. Burgers and H. van Dam, Z. Phys. C24 (1984) 247; Nucl. Phys. B260 (1985) 295.

[21] M.A.Vasiliev, Phys. Lett. B243 (1990) 378; Phys. Lett. B285 (1992) 225.

[22] M.A.Vasiliev, Phys. Lett. B567 (2003) 139, hep-th/0304049.

[23] S. W. MacDowell and F. Mansouri, Phys. Rev. Lett. 38 (1977) 739.

[24] K. Stelle and P. West,Phys. Rev. D21 (1980) 1466;

C.Preitschopf and M.A.Vasiliev, "The Superalgebraic Approach to Supergravity", hep-th/9805127.

[25] S.E.Konstein, M.A.Vasiliev and V.N.Zaikin, JHEP 0012 (2000) 018, hep-th/0010239.

[26] M.G. Eastwood, "Higher Symmetries of the Laplacian", hep-th/0206233.

[27] S.E.Konstein and M.A.Vasiliev, Nucl. Phys. B331 (1990) 475.

[28] C.Cutler and R.Wald, Class. Quant. Grav. 4 (1987) 1267;

N. Boulanger, T. Damour, L. Gualtieri, and M. Henneaux, Nucl. Phys. B597 (2001) 127, hep-th/0007220.

[29] M.A. Vasiliev, Ann. Phys. (N.Y.) 190 (1989) 59.

[30] O.V.Shaynkman and M.A.Vasiliev, Theor.Math.Phys. 123 (2000) 683, hep-th/0003123.

[31] M.A.Vasiliev, Int.J.Mod. Phys. A6 (1991) 1115; see also hep-th/9712246.

[32] L.Brink, R.R.Metsaev and M.A.Vasiliev, Nucl. Phys. B586 (2000) 183, hep-th/0005136.

[33] R.R.Metsaev, Phys. Lett. B354 (1995) 78; Phys. Lett. B419 (1998) 49, hep-th/9802097.

[34] Y.M.Zinoviev, "On massive mixed symmetry tensor fields in Minkowski space and (A)dS", hep-th/0211233; "First Order Formalism for Mixed Symmetry Tensor Fields", hep-th/0304067.

[35] K.B.Alkalaev, O.V.Shaynkman and M.A.Vasiliev, "On the Frame-Like Formulation of Mixed-Symmetry Massless Fields in $(A) d S_{d} "$, hep-th/0311164.

[36] K.B. Alkalaev, "Two-column fields in $A d S_{d}$ ", hep-th/0311212;

P. de Medeiros, "Massive gauge-invariant field theories on spaces of constant curvature", hep-th/0311254. 